\title{
Method of TCM Prescription Recognition Based on Template Matching Algorithm
}

\author{
Hanqing Zhao ${ }^{1}$, Jie Luo ${ }^{2}$, Caifeng Liu ${ }^{1}$, Xinxia Lei ${ }^{1}$ and Zhiguo Wang ${ }^{1, *}$ \\ ${ }^{1}$ Institute of Basic Research in Clinical Medicine, China Academy of Chinese Medical Sciences, Beijing, 100700, China \\ ${ }^{2}$ College of Medical Technology, Zhejiang Chinese Medical University, Hangzhou, 310053, China \\ ${ }^{*}$ Corresponding author
}

\begin{abstract}
Objective: To explore a method to identify classical recipes of traditional Chinese medicine (TCM) from clinical prescriptions of traditional Chinese medicine (TCM). Methods: By using the template matching algorithm in the field of image processing, this paper designs a kind of computer algorithm for clinical prescription recognition of Chinese medicine by constructing the relevant database and mathematical model of TCM prescription and classical prescription of traditional Chinese medicine, and implements the algorithm with Delphi language. Results: By identifying the clinical prescriptions in TCM cases, the prescriptions contained in the prescriptions can be identified, although they are not the same as the doctors' intentions. Conclusion: The clinical prescription recognition method based on template matching algorithm is feasible, which provides a reference for the further study of TCM informationization.
\end{abstract}

Keywords-information of TCM; prescription; template matching; prescription identification; modernization of TCM

\section{INTRODUCTION}

With the development of information technology, the vast amount of information produced in the clinical course of TCM is undergoing transformation from paper storage to digital storage. The storage, delivery and treatment of TCM diagnosis and treatment information have been greatly changed. Under the background of the big data era, huge amounts of information generated in the process of human production and living which contains a large amount of knowledge is not yet clear, how to effectively use information technology means the data processing in the process of modernization of traditional Chinese medicine is an important problem to be solved .At present, most grade hospital has been installed with the hospital information management system (HIS), diagnosis and treatment process of medical records, inspection, prescribing information can be used in the form of electronic medical records and electronic prescription for digital storage, If we can use appropriate methods to identify classical prescription of traditional Chinese medicine contained in each of the clinical prescription, It means a lot to the arrangement of TCM medical cases and the knowledge mining of traditional Chinese medicine in the real world [1],This paper will try to introduce the common Match Template algorithm. in the field of image processing [2] to explore the solution of this problem.

\section{INTRODUCTION OF MATCH TEMPLATE ALGORITHM}

In the field of image processing, templates usually refers to a small image, Match Template is in a large image to find out the image of a small, Under the conditions of knowing the size, direction and image content in a small image, we can find the target in the big picture through a certain algorithm, and determine its location, This method of computer processing is the Match Template algorithm. .

Concrete thought of Match Template algorithm. is, The template $\mathrm{T}(\mathrm{M} * \mathrm{~N})$ is stacked on the graph $\mathrm{S}(\mathrm{B} * \mathrm{H})$ and then shifted, The area of the map $\mathrm{S}$ override by template named subgraph Sij ,I and $\mathrm{j}$ are the coordinates of the upper left corner of the subgraph on the graph S [3]. The translation range of template $\mathrm{T}$ in figure $\mathrm{S}$ is:

$$
\begin{aligned}
& 1<=\mathrm{I}<=\mathrm{B}-\mathrm{M} \\
& 1<=\mathrm{j}<=\mathrm{H}-\mathrm{N}
\end{aligned}
$$

In the translation range, the process of template matching is completed by comparing the similarity between $\mathrm{T}$ and $\mathrm{Sij}$, and the similarity judgment of $\mathrm{T}$ and $\mathrm{Sij}$ uses (1):

$$
\mathrm{D}(\mathrm{i}, \mathrm{j})=\sum_{\mathrm{m}=1}^{\mathrm{M}} \sum_{\mathrm{n}=1}^{\mathrm{N}}\left[S_{i j}(m, n)\right]^{2}+\sum_{\mathrm{m}=1}^{\mathrm{M}} \sum_{\mathrm{n}=1}^{\mathrm{N}} T(m, n)^{2}-2 \sum_{\mathrm{m}=1}^{\mathrm{M}} \sum_{\mathrm{n}=1}^{\mathrm{N}} S_{i j}(m, n) T(m, n)
$$

As you can see from formula 1, the first and third terms are related to figure $\mathrm{S}$, the second item in the middle is only related to template $\mathrm{T}$, as the template $\mathrm{T}$ moves on the graph $\mathrm{S}$, , the first and third value is also changing, finding the minimum value of $\mathrm{D}(\mathrm{I}, \mathrm{j})$ in all translation processes is the ultimate goal of template matching.

\section{TEMPLATE MATCHING AND TCM CliniCAL PRESCRIPTION IDENTIFICATION}

Traditional Chinese medicine accumulated huge amounts of information in the inheritance and development of thousands of years, and how to use these information effectively is an important problem in the current facing of traditional Chinese medicine, and Chinese medicine clinical prescription, as a "treatment", has very important research value. In a Chinese 
medicine clinical prescription, the basic information, diagnosis and the disease information of the patient, the composition and usage of drugs, the number of agents, and the doctor's advice are generally included. Among, the composition and usage of prescription drugs jointly express the results of syndrome differentiation and treatment by prescription physicians, which can reflect the clinical thinking and drug experience of physicians to a certain extent. In most instances, the process of syndrome differentiation and prescription, doctors will choose one or several classic prescriptions to add and subtract. If information technology can be used to automatically identify [4] all the classic prescriptions contained in the clinical prescription, It is not only better to inherit, excavate and discover the rules of TCM diagnosis and treatment, but also has important significance on the inheritance of TCM schools, disease prediction and prescription evaluation [5].

Inspired by the idea of template matching algorithm, if you can think of TCM clinical prescription as a "big picture", every classical prescription can be used as a template, the idea of template matching enables the search for countless template targets in clinical prescriptions, to realize the identification of the classical prescription contained in TCM clinical prescription. On the basis of this assumption, this paper does some research on the following aspects.

\section{RESEARCH ON TCM CLINICAL PRESCRIPTION RECOGNITION METHOD}

\section{A. Research on the Digital Storage Method of TCM Clinical Prescription and TCM Classic Prescription}

At present, although Traditional Chinese hospital information management system has been equipped in all levels of hospitals, there is still no recognized standard of TCM information storage in China, and no rules for the design of TCM database. This makes the database type and format of each system impossible to be identical. Therefore, the first step of the study is to design a data storage format for TCM clinical prescription data and TCM classic prescription based on TCM medical records and prescription specifications.

Through the analysis of prescription data characteristics, establish TCM clinical prescription recognition database which using the relational model abstract the information on the basis of a strict ontological split. a simple data structure clear, relationships, norms, the advantages of simple operation. It has the advantages of simple and clear data structure, relational specification and simple operation. Microsoft SQL Server 2012 is selected as the technical tools, a cloud-based database platform that provides enterprise-level data management with integrated data mining tools. The Microsoft SQL Server 2012 database engine provides secure and reliable storage function for relational and structured data, and this is ideal for building this database. TCM clinical prescription identification database mainly stores two aspects of information, namely TCM clinical prescription data and TCM classical prescription data. The clinical prescription data of traditional Chinese medicine mainly include: case number, prescription number, diagnosis, four diagnostic summary, drug name, drug dosage, method of use, number of dosage, doctor's advice, and prescription doctor and so on. The TCM classical prescription data mainly include: prescription name, origin, drug name, dosage of drug, method of use, effective treatment, classic treatise, etc.

TCM clinical prescription data come from patients in the actual medical treatment, so the actual content should be recorded in the database, and the input format should be according to the requirements of the ontology. For example, when entering the prescription data, the drug name should be selected from the Chinese medicine data table. Thus, the standardization of data is realized. Other data in the database can be selected from various textbooks and reference books, and input them according to the database field. And the prescription data can be identified by this standard.

\section{B. Construction of Mathematical Model of TCM Clinical Prescription}

On the basis of building the support database, it is necessary to mathematical modeling TCM clinical prescription and traditional Chinese medicine prescription. Then convert it from literature knowledge into prescription data that can be recognized by computer. According to the template matching idea, the composition of prescriptions can be regarded as a matrix of several drugs and the use of doses. The prescribed prescriptions are composed of drugs A and dose M. Among them, different drugs are respectively determined by vectors [A11 A12 A13...].The corresponding dose is the vector [M11 M12 M13...], the drug matrix A is (2).

$$
A=\left[\begin{array}{cccc}
A 11 & \text { A12 } & \text { A13 } \cdots & \text { A1n } \\
\text { A21 } & & & \\
\text { A31 } & & \ddots & \\
\vdots & & & \\
\text { Am1 } & \text { Am1 } & \text { Am2 } \cdots & \text { Amn }
\end{array}\right]
$$

Establish of dose matrix $\mathrm{M}$ is (3).

$$
\mathrm{M}=\left[\begin{array}{ccc}
\mathrm{M} 11 & \ldots & \mathrm{M} 1 \mathrm{n} \\
\vdots & \ddots & \vdots \\
\mathrm{Mm} 1 & \ldots & \mathrm{Mmn}
\end{array}\right]
$$

On the basis of it, the formula matrix can be obtained as (4).

$$
\mathrm{E}=\mathrm{A} \times \mathrm{M}
$$

\section{Design of TCM Clinical Prescription Recognition Algorithm}

The significance of prescription recognition lies in finding the classic prescription contained in the clinical prescription. So we can study the clinical thinking of doctors. Throughout ancient and modern medical records, Chinese medicine practitioners attach great importance to prescription thinking, that is, syndrome differentiation, generally, doctors often choose the most suitable prescription for compatibility addition and subtraction in a general direction. As the ancients are very concerned about the "traditional Chinese medicine is not the secret of the volume," Therefore, most ancient medical records are only labeled drugs without a dose; Although modern doctors 
have abandoned this dross, most medical records in the prescriptions are available in medicine, the study found that many physicians in the dosage is quite arbitrary, the vast majority of drugs are selected in these $3 \mathrm{~g}, 10 \mathrm{~g}, 15 \mathrm{~g}, 30 \mathrm{~g}$ [6-10], does not seem to be fully in accordance with the classic formula of the drug dosage form ratio, therefore, the algorithm in the identification strategy designed to give priority to the case of drugs exactly the same. Prescribed clinical prescription is $\mathrm{S}$, the classic prescription is $\mathrm{T}$, the algorithm steps are as follows:

1) Calculate the actual number of drug $A$ in $S$ and $T$, stipulate that the total number of drugs in $\mathrm{S}$ is $\mathrm{N}$, the total number of drugs in $\mathrm{T}$ is $\mathrm{K}$

2) Performing the dimension reduction operation, the drug set $\mathrm{A}$ and the dose set $\mathrm{M}$ are respectively transformed into a one-dimensional array $\mathrm{A}$ and a one-dimensional array $\mathrm{M}$

3) Calculate the difference $\mathrm{D}$ of $\mathrm{A}[\mathrm{i}] \in \mathrm{T}$ and $\mathrm{A}[\mathrm{i}] \in \mathrm{S}$ in the range of $0 \leq \mathrm{i} \leq \mathrm{K}$

4) Repeat the third step in the range of $0 \leq \mathrm{j} \leq \mathrm{N}$

5) To sort the difference $D$, if the minimum value is not greater than 1 , then prescription $\mathrm{S}$ contains a classic prescription T .Difference D calculation method reference formula is (5).

$$
\mathrm{D}(\mathrm{i}, \mathrm{j})=\sum_{\mathrm{m}=1}^{\mathrm{N}} \sum_{\mathrm{n}=1}^{\mathrm{K}}\left[S_{i j}(m, n)-T(m, n)\right]^{2}
$$

\section{TCM Clinical Prescription Recognition Algorithm Simple to Achieve}

According to the types and characteristics of TCM clinical prescription recognition algorithm, Use Embarcadero's RAD Studio XE8 platform for the preparation of the algorithm code and implementation, RAD is a rapid model development platform based on Delphi language, which can quickly build the prototype of the project and achieve the purpose of testing the algorithm.

\section{E. Test}

The clinical prescription recognition method of TCM is based on template matching TCM clinical prescription recognition algorithm, and through the construction of TCM clinical prescription and TCM classic prescription database, the classic prescriptions in clinical prescriptions are identified by computer software.

In the classic prescription database of TCM, 666 traditional Chinese medicine names and 512 classic prescription data were collected according to the data sources, basically covering the commonly used classic prescriptions. In the clinical database of TCM, this study randomly collected clinical prescriptions from 12 TCM clinical cases [11-21] from the database of CNKI, and then run the recognition algorithm after entering the clinical prescription database of traditional Chinese medicine to compare the results of the algorithm with that of collecting physicians Case analysis of the prescription pointed out in the name, the result is as follows:
TABLE I. TCM CLINICAL PRESCRIPTION RECOGNITION RESULTS

\begin{tabular}{|l|l|}
\hline Prescription name & Recognition name \\
\hline WU ZHU YU TANG & WU ZHU YU TANG \\
\hline YIN CHEN BAI WEI TANG & NONE \\
\hline RU FANG JIANG & $\begin{array}{l}\text { HUANG LIAN WEN DAN TANG, } \\
\text { ZHI SHI SHAO YAO SAN }\end{array}$ \\
\hline $\begin{array}{l}\text { GUA LOU SHEN XIAO SAN, JU } \\
\text { HE WAN }\end{array}$ & HUO LUO XIAO LING DAN \\
\hline SHENG SUI YI XUE TANG & DANG GUI BU XUE TANG \\
\hline WAI TAI FU LING YIN & WAI TAI FU LING YIN \\
\hline ZHEN REN YANG ZANG TANG & $\begin{array}{l}\text { FU ZI LI ZHONG TANG, LI } \\
\text { ZHONG TANG }\end{array}$ \\
\hline ZHI SHI DAO ZHI WAN & ZHI ZHU WAN \\
\hline LIU WEI DI HUANG TANG & LIU WEI DI HUANG TANG \\
\hline LIU WEI SI MIAO WAN & SI MIAO WAN \\
\hline $\begin{array}{l}\text { LIU WEI DI HUANG WAN, } \\
\text { BAN XIA SHU MI TANG }\end{array}$ & $\begin{array}{l}\text { LIU WEI DI HUANG WANM, XIA JIA FU LING } \\
\text { TANG }\end{array}$ \\
\hline KOU QIAN FANG(user-define) & $\begin{array}{l}\text { SI MIAO YONG AN TANG, JIAO } \\
\text { TAI WAN, SI WU TANG }\end{array}$ \\
\hline
\end{tabular}

\section{DISCUSSION}

It can be seen from the recognition results that the clinical prescription recognition method based on the template matching algorithm can identify some of the classic prescriptions from the clinical prescriptions, but the accuracy is still low and there are still many problems:

First, the idea of template matching requires that the name of the drug in the template need to be exactly the same as the name of the drug in that part of the prescription, but in clinical practice it is very difficult to achieve complete coverage of one prescription for many reasons. For example, in this test, Pinellas tuberous appeared, but the clinical prescriptions used by physicians are yellow millet instead of rice. In the case of incomplete drug names or incomplete drug aliquots, the algorithm cannot identify yellow millet and millet similarities and differences, so it can only be judged as inconsistent, the difference must be greater than 1 .

Second, due to the different circumstances of individual patients in clinical practice, the doctor may make a slight addition or subtraction in one or several classic prescriptions when issuing a prescription. Under the influence of the template matching strategy, if the medicine is flavored, it will not affect the recognition effect, if it is reduced taste will lead to greater differences, this situation is difficult to handle. In some cases, physicians may follow their own preferences for medications such as citrus aurantiacus with trifoliate orange fruit, alternatives to other drugs of similar potency due to lack of medicine, or medications that the physician forgot to formulate The lack of and change, these factors will interfere with the recognition accuracy.

Thirdly, after thousands years of development, many traditional Chinese medicine prescriptions have created many new classic prescriptions based on the classic prescriptions of the past lives. This will result in repetition of identification. For example, aconite soup is added on the basis of LI ZHONG TANG Come, so in the use of aconite soup recipe will certainly identify these two prescriptions. This problem is both an advantage and a disadvantage of the recognition algorithm. The advantage is that it can be used to analyze the composition of a prescription and help to understand the origin of the classic 
prescription. The disadvantage is that it causes repeated recognition, resulting in the result being doped with redundant prescriptions.

Big data, artificial intelligence and computer have played a huge role in many fields. They can improve productivity greatly, help human thinking and promote the progress of discipline change. And this combines the ancient Chinese medicine with modern information science.

The significance of this method is not only the ability to identify prescriptions, but more importantly, the relationship between prescriptions can be obtained after the completion of prescription identification. Each classic prescription contains the corresponding efficacy, indications, indications, Combination analysis can be used to excavate TCM genre and develop inheritance direction, which can evaluate the clinical prescription and even can be used to predict the efficacy of prescription. In short, the use of information technology to study traditional Chinese medicine can greatly broaden our horizons, make us come out of mind set, and many overlooked or difficult to detect laws are likely to be found with the help of computer. Only a very limited attempt has been made in the study of the methods of identifying clinical prescriptions in traditional Chinese medicine. There are still many problems to be solved.

\section{ACKNOWLEDGMENT}

This research was supported by the Scientific and technological plan of traditional Chinese medicine in Zhejiang in 2015 (2015ZA065). The authors would like to sincerely thank Professor WANG in the department for his help throughout this study, as well as the doctors in the algorithm program for helping with questionnaires.

\section{REFERENCES}

[1] LIU Baoyan, "The real world of traditional Chinese medicine clinical research paradigm ," Journal of Traditional Chinese Medicine, China ,pp.54 (6): 451-455,2013.

[2] TANG Ying, LI Qing, "A fast template matching algorithm," Journal of Computer Applications, vol. 30 (6) ,pp. 1559-1561 + 1564,2010.

[3] GAO Jun, LI Xuewei, ZHANG Jian, LU Bingheng, "An image registration algorithm based on template matching,".Journal of Xi'an Jiaotong University, vol. 43 (3) ,pp.307-311,2007.

[4] LIU Xiaofeng, REN Tingge, GAO Quanquan, etc, "Research and Practice of Prescription Intelligent Analysis System of Traditional Chinese Medicine ," Chin J Inf Tradit Chin Med, vol.14 (10) ,pp.97-99, 2007.

[5] ZHAO Hanqing, "Exploration research of clinical prescription and preevaluation system of traditional Chinese medicine," China Academy of Chinese Medical Sciences , China ,pp.24-26,2015.

[6] WANG Jian, "Professor BAI Zhaozhi's academic thought and clinical experience summary and clinical research of Chinese medicine treatment of small bowel disease," Beijing University of Chinese Medicine, China , pp. 36-45,2012.

[7] CHEN Xiaoyun, "Chen Jiali's old Chinese medicine academic thought and clinical experience," Beijing University of Chinese Medicine, China ,pp.55-56,2012.

[8] SHEN Ying, "based on medical records of ancient Chinese medicine academic ideas and clinical experience of the method of inheritance," Beijing University of Chinese Medicine, China ,pp.42-43,2016.

[9] HUANG Chunhua, "Experience of traditional Chinese medicine diagnosis and treatment: a preliminary diagnosis and treatment of Yang deficiency insomnia program construction and optimization," Guangzhou University of Chinese Medicine, China ,pp.43-49,2011.
[10] BIAN Hui, "Old Chinese medicine professor Zhou Shaohua analysis of data mining experience in the treatment of stroke ," Beijing University of Chinese Medicine, China, pp.36-41,2012.

[11] HE Guoliang, "Deceased proper way, proper medication - Brief introduction of Professor Liu Chixuan's prescription medication ," Guangzhou University of Traditional Chinese Medicine, China, vol. 01 (01),pp.10-13,1985.

[12] SUN Jiaye, WanHua, "Evacuation wind heat treatment of breast disease test," Shandong Traditional Chinese Medicine, China, vol. 35 (11) ,pp.996-1000,2016.

[13] ZHOU Dan, YANG Xiaofan, LI Xin, etc, "Experience in treating Rupu with three principles of regulating qi, removing blood stasis and expectorant," Chinese Journal of Traditional Chinese Medicine, China, vol. 31 (10),pp.4069-4072,2016.

[14] WANG Xue, ZHANG Hui, WU Min, el al, "Professor ZHANG Hui's Experience in Treatment of Chronic Aplastic Anemia from Damp and Blood Stasis," Liaoning Journal of Traditional Chinese Medicine, China, vol. 41,pp.645-646,4,2014.

[15] DING Hongping, "FENG Shilun's application of foreign experience in Poria drinking clinical," Shandong Traditional Chinese Medicine, China, vol.35 (11),pp. 981-983,2016.

[16] HE Yong, MA Kexun, GONG Min, "JIANGYangqing's Experience in Differentiating and Predigesting Chronic Diarrhea (Part one) -The Application of Wen Fa ," Mediae World Chinese Medicine, China, vol.9 (2),pp. 199-201,2014

[17] HONG Qi, HONG Mei,"Citrus aurantium Zhifu pills clinical application ,'Journal of Practical Traditional Chinese Internal Medicine, China, vol. 28 (5)pp.129-130,2014.

[18] WANF YUnong, GAO Ying, "Professor GAO Ying's treatment of vertigo cases cited," World Chinese medicine,vol.9 (9)pp.1201-1205, 2014.

[19] ZHONG Yanmei, TAN Wenjuan, DUAN Peipei, etc,"Experience in treating senile vaginitis with ZENG Qian "LIU Wei SI MIAMWAN," Liaoning Journal of Traditional Chinese Medicine, China, vol.41 (4)pp.641-642,2014.

[20] XU Lisi,CHEN Xiaohui,HE Xinhui, "He Xinhui'experience in treating oral lichen planus ,'Zhejiang Journal of Traditional Chinese Medicine, China, vol. 51 (6)pp.406-407,2016,

[21] LI Rupeng, JIN Huafeng, SHAN Hongmei ,et al, “ Analysis of MA Peizhi's treatment of insomnia medical records and experience," Sichuan Journal of Traditional Chinese Medicine, China, vol. 32 (11)pp. 1720,2014 . 